APEM
Advances in Production Engineering \& Management

Volume 10 | Number 1 | March 2015 | pp 18-26

http://dx.doi.org/10.14743/apem2015.1.189
ISSN 1854-6250

Journal home: apem-journal.org

Original scientific paper

\title{
Tool selection for rough and finish CNC milling operations based on tool-path generation and machining optimisation
}

\author{
Mwinuka, T.E. ${ }^{a}{ }^{,}{ }^{,}$Mgwatu, M.I. ${ }^{a}$ \\ a University of Dar es Salaam, Department of Mechanical and Industrial Engineering, Dar es Salaam, Tanzania
}

\begin{abstract}
A B S T R A C T
Most of CAD/CAM systems lack fully-automated process planning capabilities and depend on semi-automatic capabilities that necessitate the traditional selection of tools and cutting parameters. This paper attempts to determine proper combinations of cutting tools through the generation of tool paths and optimisation of machining parameters using an example of the CNC milling process. Several machining simulations with different combinations of tool sizes were performed using MasterCAM software. Based on these simulations, substantial variations in tool paths were observed for different tool combinations and as such the optimum tool combination could only be obtained arbitrarily. The tool paths derived from machining simulations were used to optimise machining parameters, that is, cutting speed, feed rate and depth of cut with the objective of minimising production time. In this case, an optimisation model was developed as a nonlinear programming problem and solved using extended LINGO nonlinear software. The results show that the subjectivity when selecting cutting tools can be avoided when appropriate tools are chosen alongside with the generation of a tool path within a CAD/CAM system using optimised machining parameters. As a consequence, CNC machine tools could be effectively utilised and the productivity significantly improved at shorter production time and cost.
\end{abstract}

\begin{tabular}{l} 
A R T I C L E I N F O \\
\hline Keywords: \\
CAD/CAM \\
Milling operations \\
Machining optimisation \\
Nonlinear programming \\
Tool-path generation \\
Tool selection \\
*Corresponding author: \\
tmwinuka@udsm.ac.tz \\
(Mwinuka, T.E.) \\
Article history: \\
Received 30 December 2013 \\
Revised 26 December 2014 \\
Accepted 25 January 2015
\end{tabular}


APEM

\title{
Izbira orodij za grobe in fine CNC-obdelave pri rezkanju na osnovi generiranja poti orodij in optimizacije obdelave
}

\author{
Mwinuka, T.E. ${ }^{a,}{ }^{*}$, Mgwatu, M.I. ${ }^{a}$ \\ a University of Dar es Salaam, Department of Mechanical and Industrial Engineering, Dar es Salaam, Tanzania
}

\section{POVZETEK}

Velika večina CAD/CAM sistemov nima vgrajene možnosti avtomatskega načrtovanja procesa obdelave, zato se mora uporabnik še vedno posluževati tradicionalne izbire orodij in parametrov odrezovanja. Prispevek opisuje izbiro pravilnih kombinacij obdelovalnih orodij skozi generacijo poti orodij in optimizacijo obdelovalnih parametrov na zgledu CNC-rezkanja. S pomočjo orodja MasterCAM je bilo izvedenih več simulacij obdelave z različnimi orodji. Na osnovi simulacij je bilo ugotovljeno, da se poti orodij za različna orodja bistveno razlikujejo, zato je izbira optimalne kombinacije orodij prepuščena subjektivnemu odločanju. Poti orodij, ki so bile dobljene s pomočjo simulacij, smo nato uporabili za optimizacijo obdelovalnih parametrov, tj. obdelovalne hitrosti, podajanja in globine reza, pri čemer je bil cilj minimizirati čas izdelave. Takšna postavitev problema ima nelinearen značaj, zato smo ga rešili z razširjeno LINGO programsko opremo, ki omogoča reševanje nelinearnih problemov. Rezultati so pokazali, da se lahko subjektivnosti pri izbiri rezalnih orodij izognemo, če poleg ustrezne izbire orodij generiramo tudi poti orodij znotraj CAD/CAM sistema s pomočjo optimalnih parametrov odrezovanja. Predlagan pristop omogoča, da so CNC-stroji učinkoviteje izrabljeni ob hkratni povečani produktivnost, kar vodi k skrajšanju izdelovalnega časa in stroškov.

\section{PODATKI O ČLANKU}

Ključne besede:

CAD/CAM

Rezkanje

Optimizacija odrezovanja Nelinearno programiranje

Generiranje poti orodij

Izbira orodij

*Kontaktna oseba:

tmwinuka@udsm.ac.tz

(Mwinuka, T.E.)

Zgodovina članka:

Prejet 30. decembra 2013

Popravljen 26. decembra 2014

Sprejet 25. januarja 2015 


\section{References}

[1] ElMaraghy, H.A. (1993). Evolution and future perspectives of CAPP, CIRP Annals - Manufacturing Technology, Vol. 42, No. 2, 739-751, doi: 10.1016/S0007-8506(07)62537-2.

[2] Ahmad, N., Haque, A.F.M., Hasin, A.A. (2001). Current trend in computer aided process planning, In: Proceedings of the $7^{\text {th }}$ Annual Paper Meet and $2^{\text {nd }}$ International Conference, The Institution of Engineers, Bangladesh Mechanical Engineering Division, Dhaka, Bangladesh, 81-92.

[3] Tolouei-Rad, M., Payeganeh, G. (2006). A hybrid approach to automatic generation of NC programs, Journal of Achievements in Materials and Manufacturing Engineering, Vol. 14, No. 1-2, 83-89.

[4] Hou, M., Faddis, T.N. (2006). Automatic tool path generation of a feature-based CAD/CAPP/CAM integrated system, International Journal of Computer Integrated Manufacturing, Vol. 19, No. 4, 350-358, doi: 10.1080/ 09511920500504354.

[5] Prajapati, R., Rajurkar, A., Chaudhary, V. (2013). Tool path optimization of contouring operation and machining strategies for turbo machinery blades, International Journal of Engineering Trends and Technology, Vol. 4, No. 5, 1731-1737.

[6] Yao, Z., Gupta, S.K., Nau, D.S. (2001). A Geometric algorithm for finding the largest milling cutter, Journal of Manufacturing Processes, Vol. 3, No. 1, 1-16, doi: 10.1016/S1526-6125(01)70029-1.

[7] D’Souza, R., Wringt, P., Séquin, C. (2001). Automated microplanning for 2.5-D pocket machining, Journal of Manufacturing Systems, Vol. 20, No. 4, 228-296, doi: 10.1016/S0278-6125(01)80048-0.

[8] Lim, T., Corney, J., Ritchie, M., Clark, D.E.R. (2001). Optimizing tool selection, International Journal of Production Research, Vol. 39, No. 6, 1239-1256, doi: 10.1080/00207540010020063.

[9] You, C.F., Sheen, B.T., Lin, T.K. (2007). Selecting optimal tools for arbitrarily shaped pockets, The International Journal of Advanced Manufacturing Technology, Vol. 32, No. 1-2, 132-138, doi: 10.1007/s00170-005-0320-6.

[10] Mansor, M.S.A., Hinduja, S., Owodunni, O. (2006). Voronoi diagram-based tool path compensations for removing uncut material in 21/2D pocket machining, Computer-Aided Design, Vol. 38, No. 3, 194-209, doi: 10.1016/ j.cad.2005.09.001.

[11] Lee, K., Kim, T.J., Hong, S.E. (1994). Generation of toolpath with selection of proper tools for rough cutting process, Computer-Aided Design, Vol. 26, No. 11, 822-831, doi: 10.1016/0010-4485(94)90096-5.

[12] Elmesbahi, A., Rechia, A., Jaider, O. (2014). Optimized-automated choice of cutting tool machining manufacturing features in milling process, In: Proceedings of the 11th World Congress on Computational Mechanics, Barcelona, Spain, 1747-1761.

[13] Gjelaj, A., Balič, J., Ficko, M. (2013). Intelligent optimal tool selections for CNC programming of machine tools, Transactions of Famena, Vol. 38, No. 3, 31-40.

[14] Narooei, K.D., Ramli, R. (2014). Application of artificial intelligence methods of tool path optimization in CNC machines: A review, Research Journal of Applied Sciences, Engineering and Technology, Vol. 8, No. 6, 746-754.

[15] Daneshmand, S., Mirabdolhosayni, M., Aghanajafi, C. (2013). Sifting through the optimal strategies of time-based tools path machining in software CAD-CAM, Middle-East Journal of Scientific Research, Vol. 13, No.7, 844-849.

[16] Kariuki, L.W., Ikua, B.W., Nyakoe, G.N. (2014). Generation and optimization of pocket milling tool paths - A review, In: Proceedings of 2014 International Conference on Sustainable Research and Innovation, Nairobi, Kenya, Vol. 5, 129-133.

[17] LINDO Systems Inc. (2008). LINDO User's Guide, Chicago, Illinois, USA.

[18] Shunmugam, M.S., Reddy, S.V.B., Narendran, T.T. (2000). Selection of optimal conditions in multi-pass face-milling using a genetic algorithm, International Journal of Machine Tools and Manufacture, Vol. 40, No. 3, 401-414, doi: 10.1016/S0890-6955(99)00063-2.

[19] Wang, P., Liang, M. (2005). An integrated approach to tolerance synthesis, process selection and machining parameter optimization problems, International Journal of Production Research, Vol. 43, No. 11, 2237-2262, doi: $10.1080 / 00207540500050063$. 